\title{
Opposing effects of stimulus-driven and memory-driven attention in visual search
}

\author{
Koeun Jung ${ }^{1} \cdot$ Suk Won Han $^{1} \cdot$ Yoonki Min ${ }^{1}$ \\ Published online: 24 June 2019 \\ (C) The Psychonomic Society, Inc. 2019
}

\begin{abstract}
When one searches for a specific target in a cluttered visual scene, a perceptually salient stimulus or a stimulus that matches working memory's contents is prioritized for attentional selection. In the present study, we aimed at clarifying under which circumstance stimulus-driven attention or memory-driven attention is more pronounced. We hypothesized that one crucial factor affecting stimulus-driven versus memory-driven attention is how a concurrent visual search task is performed. To address this issue, we employed two visual search tasks whose underlying mechanisms are known to be different: Landolt-C search and orientation feature search. One group of participants performed visual search tasks containing a memory-matching stimulus, and the other group conducted searches in the presence of a salient singleton distractor. The results showed that the effects of stimulus-driven and memory-driven attention differed, depending on the cognitive mechanisms underlying the visual search tasks. A memory-matching stimulus captured attention when participants performed the Landolt-C search, whereas this capture was diminished under feature search. In contrast, capture by the salient singleton distractor was found only under feature search. These results demonstrate that the nature of the underlying visual search tasks is an important factor for observing stimulus-driven versus memory-driven attention. Our results also provide a potential solution to resolve current debate regarding memory-driven attention in visual search.
\end{abstract}

Keywords Stimulus-driven attention $\cdot$ Memory-driven attention $\cdot$ Visual search $\cdot$ Attentional control

When we look for a prespecified target in a complex visual scene, multiple stimuli compete for neural representation (Desimone \& Duncan, 1995). According to the biasedcompetition theory of selective attention (Desimone \& Duncan, 1995), this competition is resolved in several ways. First, among the stimuli competing against each other, a salient stimulus tends to be the winner of the competition (Beck $\&$ Kastner, 2005). Second, when a stimulus matches information maintained in working memory, competition is biased

Suk Won Han and Yoonki Min contributed equally to this work.

Electronic supplementary material The online version of this article (https://doi.org/10.3758/s13423-019-01630-9) contains supplementary material, which is available to authorized users.

Suk Won Han

suk.w.han@gmail.com

Yoonki Min

ykmin@cnu.ac.kr

1 Department of Psychology, Chungnam National University, Daejeon, Korea toward the memory-matching stimulus (Chelazzi, Duncan, Miller, \& Desimone, 1998).

In line with this theory, ample evidence has been found that a salient stimulus, even though it is task-irrelevant, captures attention (Theeuwes, 1992, 2004). However, a recent study, along with many other previous studies (Bacon \& Egeth, 1994; Barras \& Kerzel, 2017; Gaspelin, Ruthruff, \& Lien, 2016; Lamy \& Egeth, 2003), has shown that capture of attention by a salient stimulus does not always take place; the nature of the search task was found to be critical for observing stimulus-driven attentional capture (Jung, Han, \& Min, 2019). In this study, when participants performed the orientation feature search task, a singleton distractor captured attention, whereas the salient distractor failed to capture attention under the Landolt-C search task. These results were interpreted as revealing that how a search is performed determines whether or not a singleton distractor captures attention.

Specifically, even though both the Landolt-C search and the feature search were done in similarly inefficient manners, yielding significant search display set-size effects, the two searches were known to be done in differential manners: Landolt-C search proceeds in a serial, capacity-limited 
manner, heavily straining attentional control (Woodman \& Luck, 1999, 2003), whereas the feature search is presumed to be performed in a parallel manner, without recruiting much attentional resource (Huang \& Pashler, 2005; Scharff, Palmer, \& Moore, 2011).

In a previous study, Huang and Pashler (2005) employed the simultaneous-sequential paradigm, in which search stimuli were presented in two different ways: simultaneous or sequential presentation. Although the search task was done in an inefficient manner, yielding significant set-size effects, performance of the feature search task did not benefit from the sequential presentation. This result suggests that the feature search task was done in a capacity-unlimited manner. Furthermore, Palmer and colleagues showed that difficult feature searches can produce significant, steep search slopes, due to the presence of statistical decision noise (Palmer, 1994; Palmer, Ames, \& Lindsey, 1993; Palmer \& Wright, 1998). This difference in search mechanisms was suggested to be a critical factor in observing stimulus-driven attentional capture in visual search.

As with a singleton stimulus, a memory-matching stimulus was also found to capture attention in visual search. However, the effect of this search mechanism on the capture of attention by memory-matching stimuli has not been explicitly assessed. To address this issue, in the present study we tested whether memory-driven attentional capture is also dependent on how a given search is performed. As in our previous study (Jung et al., 2019), we employed two different visual search tasks whose underlying processes are known to be different: Landolt-C search and orientation feature search.

In the experiment, participants performed a visual search task with three levels of search efficiency, in the presence or absence of an attention-capturing distractor. For one group of participants (memory-driven attention group), a working memory sample - a colored geometric shape - was presented. These participants were required to memorize the shape of the memory sample. Hence, the shape of the memory sample was task-relevant, while the color was task-irrelevant. Then, a visual search task followed. In a half of the trials, a memorymatching stimulus was presented. Importantly, however, the exact same memory stimulus never appeared in the visual search display; the memory-matching distractor matched only in the task-irrelevant dimension, rendering the memorymatching stimuli completely irrelevant to the search and memory tasks (Gao et al., 2016; Soto \& Humphreys, 2009). This was done to prevent participants from strategically attending to the memory-matching stimuli during the search. Among the participants in the memory-driven attention group, half performed the Landolt-C search task, and the other half did the feature search task.

For another group (the stimulus-driven attention group), no working memory task was given. Instead, half of the total trials of visual search had a salient singleton distractor. The participants in the stimulus-driven attention group also performed either the Landolt-C search or the feature search task. Importantly, several studies have provided evidence that the search efficiency/difficulty of the concurrent visual search task is a crucial factor for observing stimulus-driven attentional capture (Barras \& Kerzel, 2017; Gaspelin et al., 2016; Jung et al., 2019; Theeuwes, 2004). To investigate whether task difficulty exerts similar effects on memory-driven attention and to control for the difference in search difficulty across search tasks, we manipulated the task difficulty.

To predict the results, if memory-driven attention exerts the same effect as stimulus-driven attention, the memorymatching distractor should interfere with target processing only under the orientation feature search task. This prediction is based on previous studies showing that both stimulusdriven and memory-driven attention are dependent on the task demands (Dalvit \& Eimer, 2011; Kiss, Grubert, Petersen, \& Eimer, 2012). According to these studies, attentional capture was strong in both stimulus-driven and memory-driven search when the task demands were low-that is, when the search display was presented until response. However, when the task demands were high, due to the brief presentation of the visual search displays, attentional capture by the salient distractors was inhibited, and the magnitude of memory-driven attentional capture also decreased. These results imply that the effect of memory-driven attention is dependent on the search demands, similar to that of stimulus-driven attention.

Alternatively, memory-driven attention might yield different results from stimulus-driven attention (Han, 2018): Memory-driven attention might be immune to the search mechanism or to search efficiency. If so, any distractor matching the working memory contents might exert interference, regardless of the type of search task.

\section{Method}

\section{Participants}

A total of 80 adults (ages $18-32$ years; 43 female, 37 male) participated in the study for monetary compensation. The sample size was estimated on the basis of our previous study showing different patterns of stimulus-driven attentional capture, depending on the search mechanism (Jung et al., 2019). All participants gave informed consent and had normal color vision and normal or corrected-to-normal visual acuity. All experimental procedures were approved by the Chungnam National University Institutional Review.

\section{Stimuli and apparatus}

The experiment was programmed and run using PsychoPy (Peirce, 2007). The stimuli were presented on a 21 -in. LCD 
monitor with a black background. The viewing distance was set to about $60 \mathrm{~cm}$. A group of participants performed a dual task, consisting of a working memory task and a visual search task (memory-driven attention group), whereas the other performed only a visual search task (stimulus-driven attention group). Each group was further divided into two groups, depending on the search task performed (see below)

The memory sample was a colored shape (star, square, triangle, hexagon, or a circle) presented at the center of the screen. The color of each shape was randomly selected from a pool of five colors (red, blue, green, yellow, or pink), each of which was produced by RGB permutations. For the participants who performed only the visual search task, no memory sample was provided (see the Design and Procedure section).

For visual search tasks, we utilized two different search tasks: Landolt-C search and orientation feature search tasks (Jung et al., 2019). In the Landolt-C search task, the search display had four outlined squares $\left(1.5^{\circ} \times 1.5^{\circ}\right)$ with a gap. The target had the gap on either the right or the left side of the square, while the distractors had the gap on either the top or the bottom side of the square. The size of the gap was set to $0.3^{\circ}, 0.6^{\circ}$, or $0.9^{\circ}$. In the feature search task, the search display contained four lines, whose length was $1.0^{\circ}$ of visual angle. The target was a line tilted to the right or the left, while distractors were vertical lines. The tilt of the target was either $4.5^{\circ}, 9^{\circ}$, or $12^{\circ}$.

\section{Design and procedure}

As we noted above, both the memory-driven and stimulusdriven attention groups were further divided into two subgroups, depending on the search task performed. Half of the participants in the memory-driven attention group performed the Landolt-C search task while maintaining a memory sample in working memory. As is shown in Fig. 1, a trial started with a 500-ms memory sample presentation. Participants were required to memorize the shape of the memory sample while ignoring its color. After a blank interval of $500 \mathrm{~ms}$, during which only the fixation dot was presented, the visual search task display was presented. The search target was an outlined square with a right or a left gap, while the distractors were squares with a top or bottom gap. Participants were required to indicate whether the gap of the target was on the right or the left. All of the search stimuli had distinct colors. The search display remained until participants had made a response (within $5,000 \mathrm{~ms}$ ). For the other half of the participants in the memory-driven attention group, the orientation feature search task was presented, in which the participants looked for a right- or left-tilted line among vertical lines and were required to report the orientation of the tilted line. Importantly, in the memory-driven attention experiments, in a half of the total trials, one of the distractors had the same color as the memory sample. We expected that this memory-matching stimulus should be attended. Hence, these trials containing a stimulus that captured attention are referred to as attended trials, in a memory-driven manner. The remaining trials were neutral ones, in which the color of the memory sample did not match any color in the search display. Following the visual search task, a memory test item was presented until participants indicated whether or not the shape of the test stimulus was the same as the shape of the memory sample (within 5,000 ms).

Participants in the stimulus-driven attention group were also divided into two subgroups, such that half of the participants in this group performed the Landolt-C search task, and the remaining half performed the orientation feature search task. Unlike the memory-driven attention group, no working memory task was presented. Importantly, in half of the total trials, a distinct color distractor was presented among gray search items. Given that these trials included a salient stimulus capturing attention, we refer these as attended trials, in a stimulus-driven manner. For each distractor-present trial, the color of the singleton distractor was randomly selected from a pool of five colors (red, yellow, green, orange, and cyan). In the remaining half, no such a singleton distractor was presented (neutral trials).

All together, the experimental design consisted of a $2 \times$ $3 \times 2 \times 2$ mixed design with distractor type (neutral vs. attended) and search difficulty (three levels, depending on gap size or target tilt) as within-subjects factors, and attention type (memory-driven vs. stimulus-driven) and search task (Landolt-C vs. feature) as between-subjects factors. For each group, participants performed six blocks, each of which included 96 trials (memory-driven experiment) or 192 trials (stimulus-driven experiment). The search difficulty level varied between blocks. Each search difficulty level had two blocks, and the block order was randomized across participants. Prior to the main experimental session, each participant performed around 10-32 practice trials to become familiar with the task.

\section{Results}

The results for the memory-driven and stimulus-driven attention groups are shown in Fig. 2 and Tables 1 and 2 . Mean search accuracy rates are summarized in Table 2 . The search accuracy rates for both groups were high and did not differ across the search trial types. To analyze visual search reaction time (RT) data, only trials with correct search responses and memory responses were used. A mixed-factor analysis of variance (ANOVA) with distractor type and search difficulty as within-subjects factors and attention type and search task as betweensubjects factors was applied to the RT data. The full details of the ANOVA results are presented in Table 1 . Given the significant four-way interaction between 
Stimulus-driven attention

(a)

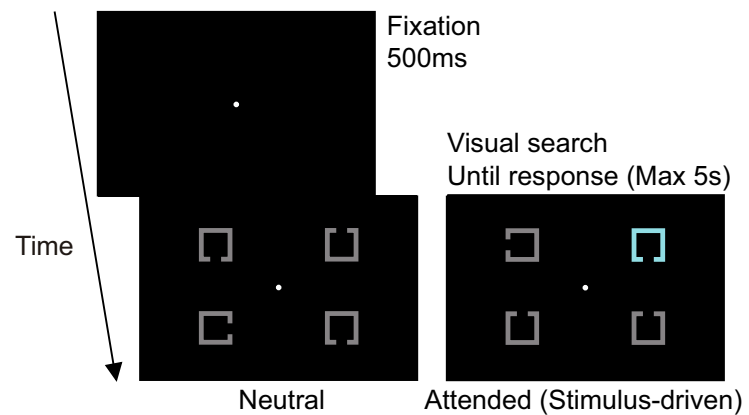

(b)

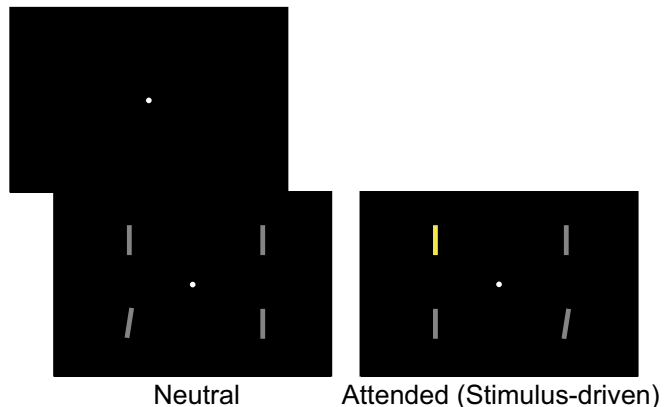

Memory-driven attention

(c)

(d)
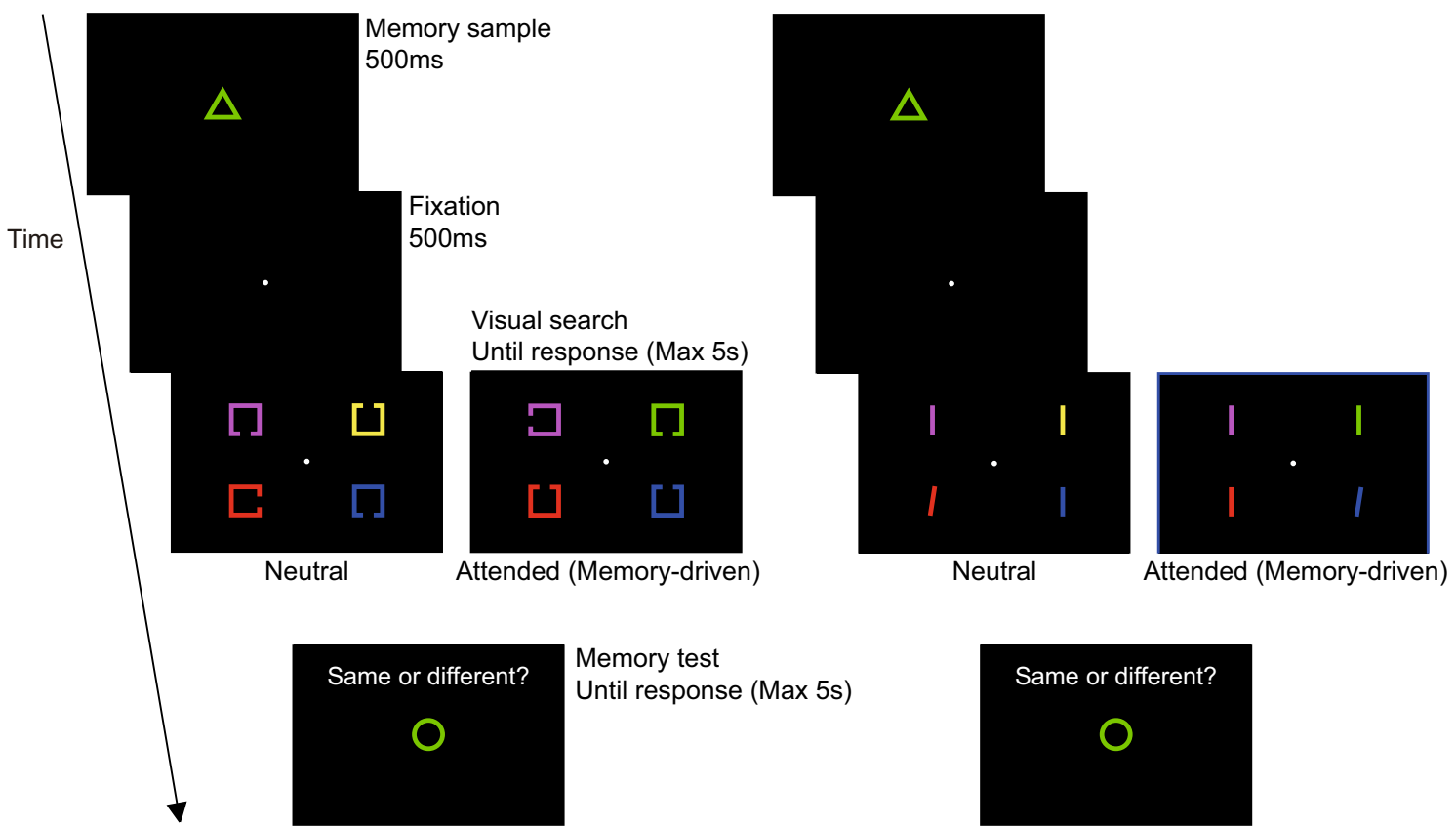

Memory test Until response (Max 5s)

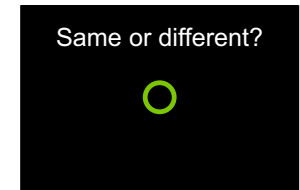

Fig. 1 Examples of experimental trials. The top panels show stimulus-driven attention [Landolt-C search task (a), orientation feature search task (b)], and the bottom panels show memory-driven attention [Landolt-C search task (c), orientation feature search task (d)]

attention type, search task, distractor type, and search difficulty, we separately examined the capture effects by the attention-capturing distractors for the different attentional types.

For the stimulus-driven attention group, a mixed ANOVA with distractor type and search difficulty as within-subjects factors, and search task as a between-subjects factor, revealed significant main effects of both search difficulty, $F(2,76)=$ 134.77, $p<.001, \eta^{2}=.78$, and distractor type, $F(1,38)=$ $10.519, p=.002, \eta^{2}=.22$. The interaction between search task and search difficulty was significant, $F(2,76)=58.07$, $p<.001, \eta^{2}=.60$. The interaction between search task and distractor type was also significant, $F(1,38)=6.353, p=.016$, $\eta^{2}=.14$. Finally, the three-way interaction was significant, $F(2,76)=11.198, p<.001, \eta^{2}=.23$.
Given the significant three-way interaction, the data were split into the Landolt-C and feature search data, and two-way ANOVAs were applied for each dataset, with distractor type and search difficulty as factors. Under the Landolt-C search task, while the main effect of search difficulty was significant, $F(2,38)=13.75, p<.001, \eta^{2}=.42$, the main effect of distractor type was not. The interaction between search difficulty and distractor type was significant, $F(2,76)=3.952, p=$ $.028, \eta^{2}=.17$.

In line with the nonsignificant main effect of distractor type, subsequent pairwise $t$ tests revealed that across all difficulty levels, the presence of the singleton distractor did not affect the Landolt-C search RTs, $p$ s $>.058$. The significant two-way interaction between distractor type and search difficulty emerged because RT patterns were 
Stimulus-driven attention

(a)

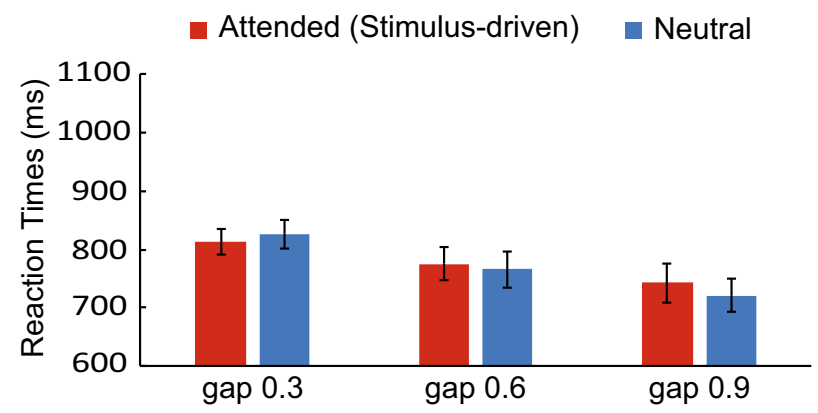

Memory-driven attention

(c)

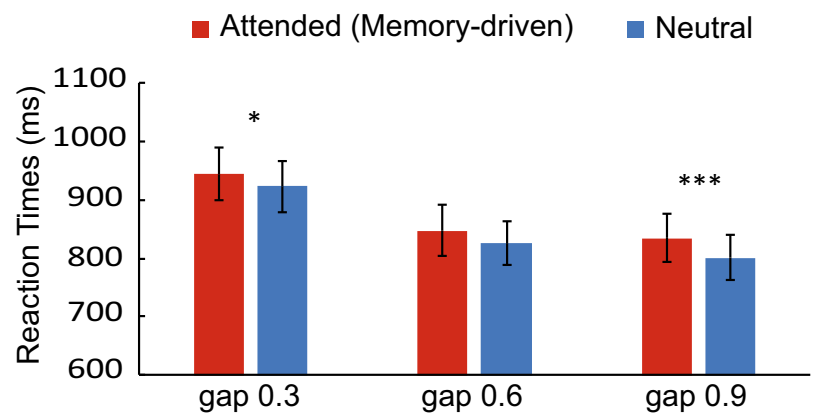

Fig. 2 Search reaction times of the experiment. The top panels show stimulus-driven attention [Landolt-C search task (a), orientation feature search task (b)], and the bottom panels show memory-driven attention

reversed as search difficulty increased, with no significant simple effect. These results show that the salient distractor (b)

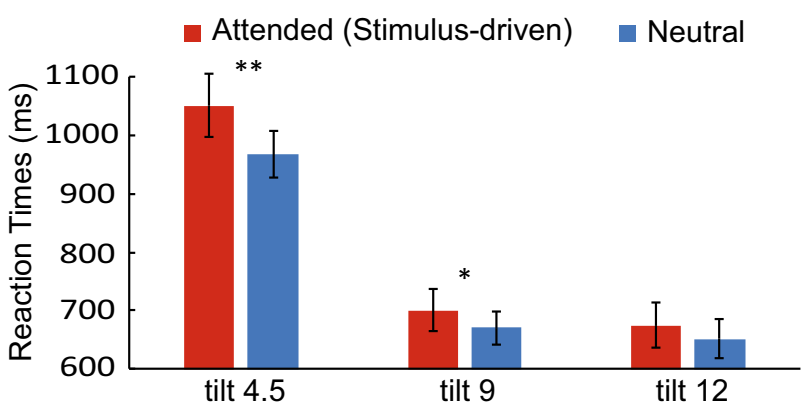

(d)

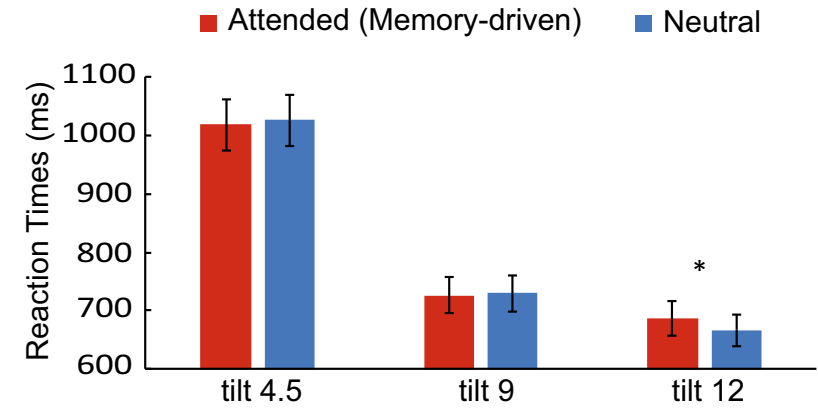

[Landolt-C search task (c), orientation feature search task (d)]. Error bars show the standard errors of the means

did not capture attention under the Landolt-C search, replicating our previous findings (Jung et al., 2019)

Table 1 Four-way mixed ANOVA results for the reaction time data of the stimulus- and memory-driven attention groups

\begin{tabular}{lll}
\hline Factors & $F$ & $p$ \\
\hline Attention type & 2.737 & .102 \\
Search task & 0.385 & .537 \\
Distractor type & 17.996 & $<.001$ \\
Search difficulty & 261.137 & $<.001$ \\
Attention type $\times$ Search task & 0.945 & .334 \\
Attention type $\times$ Distractor type & 1.425 \\
Attention type $\times$ Search difficulty & 0.243 \\
Search task $\times$ Distractor type & 0.814 \\
Search task $\times$ Search difficulty & 85.857 \\
Distractor type $\times$ Search difficulty & 0.823 \\
Attention type $\times$ Search task $\times$ Search difficulty & 1.715 \\
Attention type $\times$ Search task $\times$ Distractor type & 11.036 \\
Attention type $\times$ Distractor type $\times$ Search difficulty & 2.370 \\
Search task $\times$ Distractor type $\times$ Search difficulty & .236 \\
Attention type $\times$ Search task $\times$ Distractor $\times$ Search difficulty & 4.562 \\
\hline
\end{tabular}

$N=80$ 
Table 2 Mean search accuracy data for the stimulus- and memory-driven attention groups

\begin{tabular}{|c|c|c|c|c|}
\hline \multirow[t]{3}{*}{ Search task and search difficulty } & \multicolumn{2}{|c|}{ Stimulus-driven attention } & \multicolumn{2}{|c|}{ Memory-driven attention } \\
\hline & \multicolumn{2}{|c|}{ Task-irrelevant distractor } & \multicolumn{2}{|c|}{ Task-irrelevant distractor } \\
\hline & Present & Absent & Present & Absent \\
\hline \multicolumn{5}{|l|}{ Landolt-C Search } \\
\hline Gap 0.3 & $98.69 \%$ & $98.59 \%$ & $98.18 \%$ & $98.65 \%$ \\
\hline Gap 0.6 & $98.67 \%$ & $98.41 \%$ & $98.54 \%$ & $98.13 \%$ \\
\hline Gap 0.9 & $97.89 \%$ & $98.54 \%$ & $99.27 \%$ & $99.53 \%$ \\
\hline \multicolumn{5}{|l|}{ Feature search } \\
\hline Tilt 4.5 & $97.21 \%$ & $96.30 \%$ & $97.81 \%$ & $97.40 \%$ \\
\hline Tilt 9 & $97.13 \%$ & $96.33 \%$ & $98.59 \%$ & $98.54 \%$ \\
\hline Tilt 12 & $97.00 \%$ & $95.73 \%$ & $98.18 \%$ & $97.81 \%$ \\
\hline
\end{tabular}

Contrary to the results for the Landolt-C search dataset, under feature search the task-irrelevant distractor captured attention, as was shown by the significant main effect of distractor type, $F(1,19)=10.7, p=.004, \eta^{2}=.36$. The main effect of search difficulty was also significant, $F(2,38)=$ $139.8, p<.001, \eta^{2}=.88$. Furthermore, the interaction between distractor type and search difficulty was also significant, $p<$ .002 . Subsequent pairwise $t$ tests revealed that with the tilts of $4.5^{\circ}$ and $9^{\circ}$, the presence of singleton significantly slowed the search process, $p \mathrm{~s}<.05$. These results are consistent with previous findings of ours (Jung et al., 2019) and work by Barras and Kerzel (2017).

The results of the stimulus-driven attention group showed that the task-irrelevant singleton distractor captured attention when participants performed the orientation feature search task. Furthermore, the capture effect increased as the search efficiency decreased, replicating previous findings (Barras \& Kerzel, 2017). Unlike the feature search task, the capture by singleton distractor was not found under the Landolt- $\mathrm{C}$ search task. These results suggest that the cognitive mechanism underlying search task and search efficiency affect stimulusdriven attentional capture (Jung et al., 2019).

We applied the same analysis to the memory-driven attention group data. The analysis revealed significant main effects of search difficulty, $F(2,76)=127.28, p<.001, \eta^{2}=.77$, and distractor type, $F(1,38)=7.804, p=.008, \eta^{2}=.17$. The twoway interactions between search task and search difficulty, $F(2,76)=31.82, p<.001, \eta^{2}=.46$, and between search task and distractor type, $F(2,76)=4.916, p=.033, \eta^{2}=.12$, were significant. The three-way interaction was not significant, $p>$ .77. Despite the lack of a significant three-way interaction, we separately examined the Landolt-C and feature search data, for the following two reasons. First, the significant two-way interaction between search task and distractor type necessitated an examination of the capture effects in different tasks. Second, several literatures in memory-driven attentional capture have shown that the task demands or the nature of the visual search task employed were critical to observing the capture effect (Dalvit \& Eimer, 2011; Olivers, 2009).

For both search tasks data, we applied two-way ANOVAs of distractor type and search difficulty. Under Landolt-C search, significant main effects of distractor type, $F(1,19)=$ $18.45, p<.001, \eta^{2}=.49$, and search difficulty, $F(2,38)=$ $30.73, p<.001, \eta^{2}=.62$, were found. The interaction between distractor type and search difficulty was not significant, $p>$ .63. These results suggest that when participants performed the Landolt-C search task, a memory-matching stimulus captured attention, interfering with target search.

Under orientation feature search, only the main effect of search difficulty was significant, $F(2,38)=96.6, p<.001, \eta^{2}$ $=.84$. Neither the main effect of distractor type nor the interaction between the factors was significant, $p \mathrm{~s}>.18$. These results indicate that no memory-driven attention was found under feature search. However, notably, previous studies have shown significant memory-driven attention in settings similar to that of the present study (Gao et al., 2016; Soto \& Humphreys, 2009). In these studies, memory-driven attentional capture was found when the target tilt was $12^{\circ}$. Hence, we also examined the results of the trials, in which the target tilt was $12^{\circ}$. A pairwise $t$ test showed that search RTs were significantly longer for the memory-matching distractor trials than for the neutral trials, $t(19)=2.142, p<.05$, consistent with the results from Gao and colleagues and Soto and Humphreys. Hence, we do not make a strong claim that memory-driven attention does not take place under feature search. However, it is clear that the extent to which a memory-matching stimulus captures attention is greater under Landolt-C search than under feature search.

To clarify whether the observed memory-driven capture effect was due to memory-driven attention or bottom-up priming, we ran control experiments ( $N=20$ for each search task). These experiments were identical to the memory-driven attention conditions, except that participants were not required to maintain the memory item in working memory for a later 
memory test. The search RT analysis showed that there was no effect of priming under the Landolt-C search task, $F(1,19)=$ $0.206, p=.655$, or under the feature search task, $F(1,19)=$ $0.148, p=.705$ (see Fig. 3). These results indicate that the presently observed effect is clearly driven by working memory.

Taken together, for both the memory-driven and stimulusdriven attention groups, attentional capture by the taskirrelevant distractor was different across the search tasks. However, the specific patterns of the capture were opposite. In stimulus-driven attention, capture by the task-irrelevant distractor was observed only under the feature search task, and this capture increased as search efficiency decreased (Barras \& Kerzel, 2017; Jung et al., 2019). On the contrary, the task-irrelevant distractor did not capture attention when participants performed the Landolt-C search. The memorydriven attention group showed a differential pattern. We found that while participants performed the Landolt-C search, a taskirrelevant (memory-matching) distractor did capture attention. However, no memory-driven attentional capture was found under feature search. The significant four-way interaction between attention type, search task, distractor type, and search difficulty confirmed this pattern of results.

\section{Discussion}

We found that the effects of stimulus-driven and memorydriven attention differed, depending on the cognitive mechanisms underlying the visual search tasks. A salient singleton distractor that captured attention in a stimulus-driven manner exerted significant interference under the orientation feature search task. By contrast, under the Landolt-C search task, the singleton distractor did not capture attention. A memorymatching distractor was found to exert an effect different from that of a singleton distractor. Specifically, under the Landolt-C search, a stimulus matching the working memory contents captured attention, interfering with the target search. By contrast, no such memory-driven attentional capture was found under the orientation feature search.

(a)

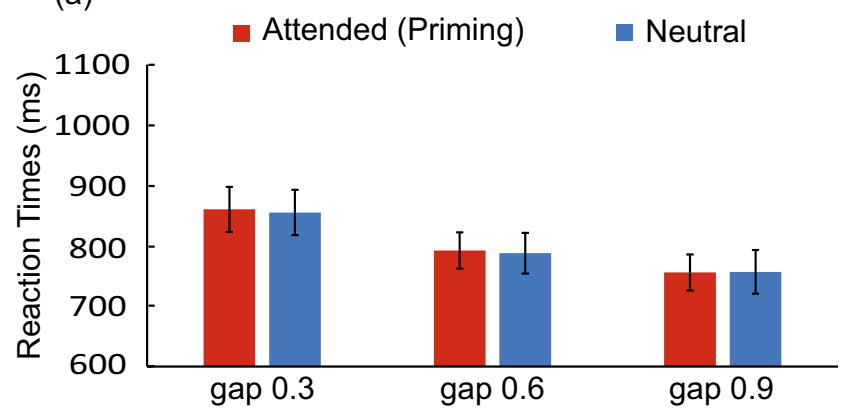

We suggest that these dissociable effects of stimulus-driven and memory-driven attention in visual search are observed because the Landolt-C search task consumes capacitylimited attentional resources, thereby straining attentional control to a greater extent than does the orientation feature search task. Notably, under this demanding search condition, the participants in the memory-driven attention group were also required to maintain an object in working memory for a subsequent memory test. Several studies have suggested that working memory and attention share a common pool of processing resource (Awh \& Jonides, 2001; Kiyonaga \& Egner, 2013). Given this, some attentional resources should already be consumed to retain the memory sample in working memory while the search proceeds. Under this competition between the two processes, the target search should be susceptible to the interference exerted by the memory-matching distractor. Unlike the Landolt-C search task, the feature search task is known to proceed without the recruitment of many attentional resources (we refer readers to the General Discussion section of Jung et al., 2019). Under this search condition, the competition between the maintenance of the memory item and visual search would be minimized.

In the stimulus-driven attention group, on the contrary, participants retained only an attentional template that represented the current search goal in order to assess incoming visual inputs as potential targets, with no accessory item in working memory. Furthermore, the target template is moved relatively rapidly from visual working memory to other memory systems when the target is constant across trials (Carlisle, Arita, Pardo, \& Woodman, 2011). In this case, the processing resources to perform a visual search need not be shared with other processes (e.g., maintaining the memory item).

Another important finding is that stimuli that match working memory, even though they match in a task-irrelevant dimension, can capture attention. Note especially that, in the present paradigm, no stimulus exactly matching the memory item was presented in the search display. While participants were required to memorize the shape of a colored geometric shape, a distractor in the search array matched the memory sample in color only. Hence, a stimulus exactly identical to the

\section{(b)}

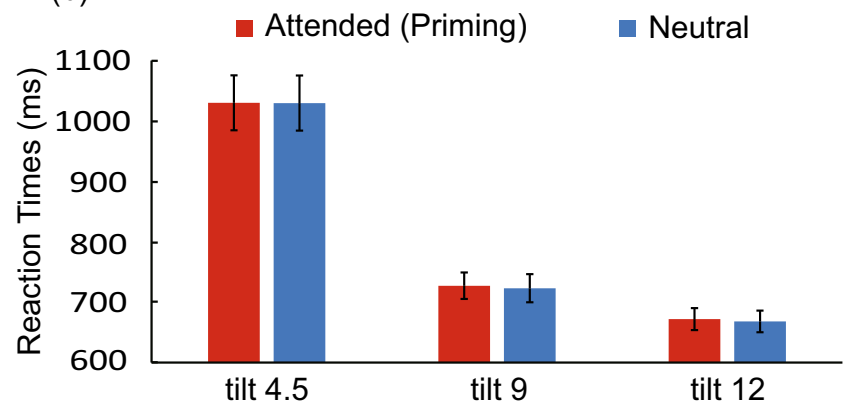

Fig. 3 Search reaction times in the control experiment: Mean reaction times for the Landolt-C search task (a) and the orientation feature search task (b). Error bars show the standard errors of the means 
memory sample never appeared in the search display. Given this fact, we are confident that there should be no incentive for strategic attending to the memory-matching stimuli. Considering this, the presently observed memory-driven attentional capture took place in an automatic manner.

The fact that the memory-matching stimuli of the present study were entirely task-irrelevant provides an adequate explanation for a discrepancy between the present study and previous studies. Some previous studies have showed that working memory can affect attentional control even in the presence of a pop-out target (Dowd \& Mitroff, 2013; Soto, Humphreys, \& Heinke, 2006) and distractor (Olivers, Meijer, \& Theeuwes, 2006). However, no memory-driven attentional capture was found under feature search in the present study. In the previous studies, the memory-matching stimuli the memory sample in the task-relevant feature. In the present study, on the contrary, the memory-matching stimuli matched working memory's contents in the task-irrelevant dimension only. Hence, we suggest that task relevance is a crucial factor reconciling the discrepancy. This hypothesis is supported by Bundesen (1990), who suggested that task-relevant features attract people's attention more powerfully than do task-irrelevant features.

A question could arise whether stimulus-driven and memory-driven attention can be directly compared, because the search displays from each attention group differed: Whereas stimulus-driven attention had a pop-out stimulus, the memory-matching distractor did not pop out. Although we argue that this difference was necessary, to separate the effect of a pure bottom-up factor from that of memory-driven attention, we also admit that the interaction between bottom-up and topdown factors will be an important point for future studies to investigate in order to fully elucidate the distinct natures of stimulus-driven and memory-driven attention.

To conclude, the present study showed that memory-driven attention has an effect opposed to that of stimulus-driven attention in visual search. Stimuli that matched working memory captured attention when participants performed the Landolt-C search, which heavily strained attentional control, whereas this capture was barely observable under feature search. In contrast, capture by a salient singleton distractor was found only under feature search. Our results demonstrate that the search mechanism of visual searches is an important factor in observing both stimulus-driven and memory-driven attention. These findings further suggest a tight interaction between the extent to which attentional control is strained and working memory.

Acknowledgments K.J. and Y.M. were supported by the Ministry of Science and Information \& Communications Technology, Korea, under the Information Technology Research Center support program (IITP2019-2016-0-00304), supervised by the Institute for Information \& Communications Technology Promotion. A National Research Foundation grant funded by the Korean government (NRF2019R1F1A1040940) supported S.W.H.

\section{References}

Awh, E., \& Jonides, J. (2001). Overlapping mechanisms of attention and spatial working memory. Trends in Cognitive Sciences, 5, 119-126. https://doi.org/10.1016/S1364-6613(00)01593-X

Bacon, W. F., \& Egeth, H. E. (1994). Overriding stimulus-driven attentional capture. Perception \& Psychophysics, 55, 485-496. https:// doi.org/10.3758/BF03205306

Barras, C., \& Kerzel, D. (2017). Target-nontarget similarity decreases search efficiency and increases stimulus-driven control in visual search. Attention, Perception, \& Psychophysics, 79, 2037-2043. https://doi.org/10.3758/s13414-017-1367-9

Beck, D. M., \& Kastner, S. (2005). Stimulus context modulates competition in human extrastriate cortex. Nature Neuroscience, 8,1110 1116. https://doi.org/10.1038/nn1501

Bundesen, C. (1990). A theory of visual attention. Psychological Review, 97, 523-547. https://doi.org/10.1037/0033-295X.97.4.523

Carlisle, N. B., Arita, J. T., Pardo, D., \& Woodman, G. F. (2011). Attentional templates in visual working memory. Journal of Neuroscience, 31, 9315-9322. https://doi.org/10.1523/ JNEUROSCI.1097-11.2011

Chelazzi, L., Duncan, J., Miller, E. K., \& Desimone, R. (1998). Responses of neurons in inferior temporal cortex during memoryguided visual search. Journal of Neurophysiology, 80, 2918-2940.

Dalvit, S., \& Eimer, M. (2011). Memory-driven attentional capture is modulated by temporal task demands. Visual Cognition, 19, 145153.

Desimone, R., \& Duncan, J. (1995). Neural mechanisms of selective visual attention. Annual Review of Neuroscience, 18, 193-222. https://doi.org/10.1146/annurev.ne.18.030195.001205

Dowd, E. W., \& Mitroff, S. R. (2013). Attentional guidance by working memory overrides salience cues in visual search. Journal of Experimental Psychology: Human Perception and Performance, 39, 1786-1796. https://doi.org/10.1037/a0032548

Gao, Z., Yu, S., Zhu, C., Shui, R., Weng, X., Li, P., \& Shen, M. (2016). Object-based encoding in visual working memory: Evidence from memory-driven attentional capture. Scientific Reports, 6, 22822.

Gaspelin, N., Ruthruff, E., \& Lien, M. C. (2016). The problem of latent attentional capture: Easy visual search conceals capture by taskirrelevant abrupt onsets. Journal of Experimental Psychology: Human Perception and Performance, 42, 1104-1120. https://doi. org/10.1037/xhp0000214

Han, S. W. (2018). Opposing effects of memory-driven and stimulusdriven attention on distractor perception. Cognitive Processing, 19, $117-123$.

Huang, L., \& Pashler, H. (2005). Attention capacity and task difficulty in visual search. Cognition, 94, B101-B111. https://doi.org/10.1016/j. cognition.2004.06.006

Jung, K., Han, S. W., \& Min, Y. (2019). Search efficiency is not sufficient: The nature of search modulates stimulus-driven attention. Attention, Perception, \& Psychophysics, 81, 61-70. https://doi.org/10.3758/ s13414-018-1598-4

Kiss, M., Grubert, A., Petersen, A., \& Eimer, M. (2012). Attentional capture by salient distractors during visual search is determined by temporal task demands. Journal of Cognitive Neuroscience, 24, 749-759. https://doi.org/10.3758/s13414-012-0391-z

Kiyonaga, A., \& Egner, T. (2013). Working memory as internal attention: Toward an integrative account of internal and external selection processes. Psychonomic Bulletin \& Review, 20, 228-242.

Lamy, D., \& Egeth, H. E. (2003). Attentional capture in singletondetection and feature-search modes. Journal of Experimental Psychology: Human Perception and Performance, 29, 1003-1020. https://doi.org/10.1037/0096-1523.29.5.1003

Olivers, C. N. L. (2009). What drives memory-driven attentional capture? The effects of memory type, display type, and search type. Journal 
of Experimental Psychology: Human Perception and Performance, 35, 1275-1291. https://doi.org/10.1037/a0013896

Olivers, C. N. L., Meijer, F., \& Theeuwes, J. (2006). Feature-based memory-driven attentional capture: Visual working memory content affects visual attention. Journal of Experimental Psychology: Human Perception and Performance, 32, 1243-1265. https://doi.org/10. 1037/0096-1523.32.5.1243

Palmer, J. (1994). Set-size effects in visual search: The effect of attention is independent of the stimulus for simple tasks. Vision Research, 34, 1703-1721.

Palmer, J., Ames, C. T., \& Lindsey, D. T. (1993). Measuring the effect of attention on simple visual search. Journal of Experimental Psychology: Human Perception and Performance, 19, 108-130. https://doi.org/10.1037/0096-1523.19.1.108

Palmer, J., \& Wright, R. (1998). Attentional effects in visual search: Relating search accuracy and search time. Visual Attention, 8, $348-388$.

Peirce, J. W. (2007). PsychoPy-Psychophysics software in Python. Journal of Neuroscience Methods, 162, 8-13. https://doi.org/10. 1016/j.jneumeth.2006.11.017

Scharff, A., Palmer, J., \& Moore, C. M. (2011). Extending the simultaneous-sequential paradigm to measure perceptual capacity for features and words. Journal of Experimental Psychology: Human Perception and Performance, 37, 813-833. https://doi.org/ 10.1037/a0021440

Soto, D., \& Humphreys, G. W. (2009). Automatic selection of irrelevant object features through working memory: Evidence for top-down attentional capture. Experimental Psychology, 56, 165-172.
Soto, D., Humphreys, G. W., \& Heinke, D. (2006). Working memory can guide pop-out search. Vision Research, 46, 1010-1018.

Theeuwes, J. (1992). Perceptual selectivity for color and form. Perception \& Psychophysics, 51, 599-606. https://doi.org/10.3758/ BF03211656

Theeuwes, J. (2004). Top-down search strategies cannot override attentional capture. Psychonomic Bulletin \& Review, 11, 65-70. https:// doi.org/10.3758/BF03206462

Woodman, G. F., \& Luck, S. J. (1999). Electrophysiological measurement of rapid shifts of attention during visual search. Nature, 400 , 867-869.

Woodman, G. F., \& Luck, S. J. (2003). Serial deployment of attention during visual search. Journal of Experimental Psychology: Human Perception and Performance, 29, 121-138. https://doi.org/10.1037/ 0096-1523.29.1.121

\section{Open Practices Statement}

The data for all experiments are available as electronic supplementary material, and none of the experiments was preregistered.

Publisher's note Springer Nature remains neutral with regard to jurisdictional claims in published maps and institutional affiliations. 